\title{
Promoviendo la apropiación del modelo de energía en estudiantes de 4. de ESO a través del diseño didáctico
}

\author{
Macarena Soto ${ }^{1}$, Digna Couso ${ }^{2}$, Víctor López ${ }^{2}$, María Isabel Hernández ${ }^{2}$ \\ ${ }^{1}$ Universidad de Santiago de Chile. \\ ${ }^{2}$ CRECIM-Universidad Autónoma de Barcelona
}

[Recibido el 10 de marzo de 2017, aceptado el 02 de mayo de 2017]

\begin{abstract}
Presentamos una investigación sobre la apropiación del modelo de energía por parte de estudiantes de 4ㅇ de ESO que participan de un taller experimental centrado en la modelización (ideas de transferencia, conservación y degradación de la energía), identificando los grados de sofisticación en el uso del modelo y las fronteras conceptuales que superan, y usando este análisis para orientar el refinamiento iterativo del diseño del taller (perspectiva de diseño basado en la investigación). Encontramos que la mayoría de estudiantes pasan de explicar los fenómenos en términos de transformación de energía a hacerlo en términos de transferencia, y que el grado de sofisticación de sus explicaciones es substancialmente mayor después de refinar el taller. En particular, se muestran especialmente eficientes los constructos didácticos gráficos/lingüísticos que proporcionan andamiaje en la mirada a los fenómenos des del modelo de energía.
\end{abstract}

Palabras clave: energía, investigación basada en el diseño (DBR), modelos conceptuales, modelización, progresión de aprendizaje.

\section{Promoting 4th ESO students' appropriation of energy model through the didactic design}

We present a research about the appropriation of the model of energy by 4th ESO students that participate in a modeling labwork-based workshop dealing with the ideas of energy transfer, conservation and degradation, identifying different degrees of sophistication in the use of the model of energy by students and some conceptual boundaries necessary to be crossed. We use this analysis to iteratively refine the workshop (Design-based research perspective). We find that most of students move from an energy transformation-based explanation to an energy transferbased explanation, and this improvement is substantially bigger after introducing several didactic changes in the teaching and learning proposal. In particular, graphical/linguistic didactic artifacts that scaffold students' way of looking at phenomena from the model of energy perspective show especially efficient.

Keywords: Energy, research-based design (DBR), conceptual models, modeling, learning progression.

Para citar el artículo. Soto, M., Couso, D., López, V. y Hernández, M.I. (2017). Promoviendo la apropiación del modelo de energía en estudiantes de 4. de ESO a través del diseño didáctico. Ápice. Revista de Educación Científica, 1(1), 90-106. DOI: https://doi.org/10.17979/ arec.2017.1.1.2003

Contacto.macarena.sotoa@usach.cl, digna.couso@uab.cat, victor.lopez@uab.cat, mariaisabel.hernandez@uab.cat 


\section{El reto de la enseñanza y aprendizaje de la energía en la escuela: ¿Por qué tan importante y a la vez tan difícil?}

La energía y sus diferentes conceptos asociados - los llamados energy-related concepts propuestos por Ogborn (1986), es decir su conservación, su degradación y su transferencia - configuran el que es, posiblemente, uno de los modelos científicos más centrales, potentes, fructíferos y unificadores de la ciencia (Ogborn, 1986; Solbes y Tarín, 1998; Millar, 2005; Neumann, Viering, Boone y Fischer, 2013). El amplio abanico de fenómenos y contextos en los que la ciencia recurre a estas ideas para explicar los hechos del mundo (desde las cadenas tróficas hasta el calentamiento global, pasando por el origen del universo o los terremotos), así como la variedad de disciplinas en las que estas ideas están presentes (desde la termodinámica hasta la bioquímica, pasando por el electromagnetismo o la ingeniería) dan prueba de esta centralidad. No es extraño, por lo tanto que, en la ciencia escolar la energía y sus conceptos asociados gocen también de gran interés, estando presentes en todos los currículos (Solomon, 1985; Solbes y Tarín, 2004; Pérez-Landazábal y Varela, 2006) y siendo una de las 10 grandes ideas de ciencia (Harlen, 2010). Además, la apuesta paulatina por enfoques educativos que promueven la alfabetización y la competencia científica dan todavía más sentido a la enseñanza de la energía: mirar el mundo a través de la energía ayuda a construir un pensamiento crítico y responsable, a tener herramientas para discutir y opinar sobre cuestiones socialmente relevantes como la carencia de recursos energéticos en el mundo y el mal uso que se hace de ellos, o bien sobre la necesidad de apostar como sociedad por maneras más eficientes y rentables de almacenar y transferir energía (García-Carmona y Criado, 2008, 2010).

No obstante, la misma centralidad, potencia y naturaleza unificadora de los energyrelated concepts también los hacen ser una de las ideas científicas más difíciles de enseñar y aprender de forma adecuada, hecho que resulta en un bajísimo nivel de comprensión de esta idea en los estudiantes que terminan sus estudios de secundaria (Domenech, Limiñana y Menargues, 2013). Una extensa actividad investigadora en nuestra área durante más de 40 años sobre cómo enseñar y/o a aprender energía da cuenta de esta problemática, analizando las principales dificultades y obstáculos. En la literatura se identifican como principales problemas a) la propia selección de contenidos a enseñar (Obgorn, 1986; López y Pinto, 2012a); b) las múltiples concepciones alternativas y espontáneas identificadas históricamente en los estudiantes (Duit, 1981, 1984; Driver y Warrington, 1985; Pintó, 1991; Solbes y Tarín, 1998; Pérez, Marbà e Izquierdo, 2016); c) la versión de estas mismas concepciones identificadas en el profesorado y en los materiales educativos (De Almeida Pacca y Henrique, 2004; Pintó, Couso y Gutiérrez, 2005) y d) la falta de eficacia de las propuestas tradicionales que abordan esta temática (Pérez-Landazábal y Varela, 2006). A todo esto se le añade la propia ambigüedad y confusión con la que a menudo se aborda en niveles universitarios (Doménech, Limiñana y Menargues, 2003; Alomá y Malaver, 2007), y muy especialmente las interferencias con el lenguaje cotidiano y los usos que se da del término en contextos no científicos (en la publicidad, en la psicología, e incluso en la metafísica, en la ficción y en las pseudociencias). En palabaras de Millar (2005): "no es una exageración decir que la enseñanza de la energía es un lio".

En paralelo a la investigación en torno a las dificultades de enseñanza y aprendizaje de la energía, se han elaborado distintas propuestas y aproximaciones didácticas para superarlas y conseguir así mejores resultados en el aprendizaje de la energía en la escuela. Desde el proyecto pionero Energy and Change de la Nuffield Foundation hasta 
el reciente Supporting Physics Teaching 11-14 de la británica Institut of Physics, múltiples propuestas han ofrecido recursos, contextos, ideas, actividades y ejemplos para construir un modelo escolar de energía adecuado. A pesar de ello, la discusión todavía existe dentro de la comunidad académica (solamente hay que ver como en cualquier congreso de didáctica de las ciencias el tema está presente), lo que muestra que las cuestiones centrales en el área referentes a la enseñanza del modelo escolar de energía siguen abiertas. Si bien históricamente éstas han sido qué modelo o conjunto de ideas enseñar y qué metodología didáctica desarrollar para promover su aprendizaje, en este artículo hemos querido poner el énfasis en una tercera cuestión, inspirada en la lógica de las progresiones de aprendizaje (Duschl, Maeng y Sezen, 2011). Asumiendo que el modelo escolar de energía se construye a lo largo de la escolaridad, y que los alumnos usan diferentes versiones del mismo, más o menos sofisticadas, en sus explicaciones de fenómenos en términos energéticos, nos preguntamos: 1) cuáles son las diferentes versiones del modelo de energía que los estudiantes usan y 2) cómo influye el diseño didáctico en la evolución del grado de sofisticación de las mismas.

\section{Modelos, modelización y versiones del modelo escolar de energía}

\section{Hacia un modelo científico escolar de energía útil y coherente}

Los enfoques de enseñanza de la energía más comunes presentes todavía hoy en libros y materiales didácticos se basan en ideas como "la energía ni se crea ni se destruye, solo se transforma", o bien "existen muchos tipos de energía: cinética, eléctrica, lumínica, química, calorífica, etc., y cada una tiene su fórmula". Estos han resultado francamente ineficaces a la hora de ayudar a los estudiantes a construir un modelo adecuado de energía, ya que no permiten explicar los fenómenos ni mucho menos predecir o actuar en ellos. Por ejemplo, decir que en un circuito eléctrico simple "la energía química de la pila se transforma en energía eléctrica y luego en energía lumínica" no solo no explica nada (no requiere conocer ninguno de los mecanismos ni físicos ni químicos que pasan en la pila, en el cable conductor o en el filamento de la bombilla), sino que además no permite pensar en qué momento la energía es más o menos aprovechable, qué maneras hay de ahorrar energía haciendo más eficiente el proceso o que parte del proceso es reversible y cual no, entre otras.

Si bien no existe una única manera ideal de exponer las ideas del modelo escolar de energía que no acarree alguna crítica o contradicción insalvable, años de discusión en el área (Ogborn 1986; Devi, Tiberghien, Baker y Brna, 1996; Millar, 2005; Papadouris, Constatinou y Kyratsi, 2008) nos llevan a seleccionar cuatro ideas clave que definen la mirada energética a los fenómenos (Tabla 1). Estas ideas incluyen la propia conceptualización de energía como una función de estado, así como sus tres conceptos relacionados (transferencia, conservación y degradación).

Estas cuatro ideas permiten expresar un modelo científico que debería trabajarse a lo largo de la escolaridad con el objetivo de que los estudiantes lo vayan construyendo progresivamente. 
Tabla 1. Cuatro ideas clave que definen la mirada energética a los fenómenos

\begin{tabular}{|l|l|}
\hline \multirow{2}{*}{$\begin{array}{l}\text { Idea de } \\
\text { estado }\end{array}$} & $\begin{array}{l}\text { La energía es una función de estado de los sistemas. En otras palabras, } \\
\text { asociamos energía a las cosas en función de cómo están (están calientes, } \\
\text { elevadas, comprimidas, iluminadas, agitadas, enlazadas, etc.). Puesto que } \\
\text { solamente tiene sentido definir un estado respecto a un cambio o un sistema } \\
\text { de referencia, no medimos la energía absoluta de los sistemas, sino los } \\
\text { cambios de energía que se producen en ellos cuando cambia su estado. }\end{array}$ \\
\hline $\begin{array}{l}\text { Existe transferencia de energía entre sistemas, o entre partes del sistema, } \\
\text { asociados a los cambios que experimentan los sistemas o sus partes, pero esta } \\
\text { transferencia } \\
\text { transferencia no es siempre igual: si intervienen fuerzas que causan } \\
\text { intervienen cuerpos que inicialmente se encuentran a diferente temperatura, } \\
\text { la transferencia es por calor. Así, podemos pensar en calor y trabajo como dos } \\
\text { formas o maneras diferentes de transferir energía. }\end{array}$ \\
\hline $\begin{array}{l}\text { Idea de } \\
\text { conservación }\end{array}$ & $\begin{array}{l}\text { La energía se conserva en sistemas aislados, y se disipa irremediablemente en } \\
\text { los sistemas abiertos. }\end{array}$ \\
\hline $\begin{array}{l}\text { Idea de } \\
\text { degradación }\end{array}$ & $\begin{array}{l}\text { genergía se degrada a medida que un sistema va perdiendo capacidad para } \\
\text { menos útil. }\end{array}$ \\
\hline
\end{tabular}

\section{Construir el modelo escolar de energía mediante la modelización}

Hablar del modelo escolar de energía obliga a reflexionar brevemente sobre modelos y modelización. Si bien uno de los significados más populares de la palabra modelo es el de "representación concreta de alguna cosa" (Justi, 2006), en su revisión del campo Oh y Oh (2011) señalan que no hay un consenso establecido para este término, que es enormemente polisémico en el ámbito de la enseñanza de las ciencias. Desde nuestra perspectiva, fuertemente influenciada por la corriente semanticista en filosofía de la ciencia y la perspectiva de la Actividad Científica Escolar, concebimos los modelos científicos escolares (los modelos que nos interesan en la escuela) como versiones escolarmente adecuadas de los modelos científicos (de la ciencia), que son de naturaleza teórica y conceptual, y que tienen la capacidad de describir, explicar, predecir e intervenir en el mundo (Izquierdo-Aymerich y Adúriz-Bravo, 2003; Hernández, Couso y Pintó, 2015). Como modelos teóricos son entidades abstractas, no lingüísticas, que se comportan según las proposiciones que los definen en cualquier sistema simbólico y que constituyen la "parte aplicativa de una teoría" (sus proyecciones al mundo) (Giere, 1992; Adúriz-Bravo e Izquierdo-Aymerich, 2009). Como modelos conceptuales, son representaciones externas (y por tanto no mentales) compartidas por una comunidad (en este caso la escolar o didáctica), que tienen coherencia con el conocimiento científico de referencia (en este caso las ciencias experimentales), y que han sido creadas por los miembros de la misma (docentes, investigadores en didáctica de las ciencias) para facilitar la comprensión e intervención en los fenómenos del mundo que intentan modelizar (Greca y Moreira, 2000). Aunque en la literatura en didáctica de las ciencias a menudo se establece una equivalencia entre las diferentes materializaciones de un modelo conceptual (por ejemplo las maquetas, esquemas, enunciados, formulaciones matemáticas, etc.) y el modelo conceptual en sí mismo, para nosotros el modelo conceptual hace referencia a las ideas, reglas del juego y forma de mirar subyacente a las diferentes materializaciones o expresiones del modelo, y no cada una de ellas. Por ejemplo, no consideramos que una maqueta del sistema solar sea un modelo conceptual del sistema solar, pero sí puede ser una expresión privilegiada del mismo que usada de cierta forma permita que los estudiantes se lo vayan apropiando. En este sentido, no consideramos que el modelo científico escolar de energía sean los 
enunciados de la Tabla 1, pero sí que estas son una expresión privilegiada del mismo útil para el diseño curricular y la enseñanza y aprendizaje.

Ejemplos de estos modelos científicos escolares son los modelos científicos escolares clave (o familias de modelos o teorías científicas escolares), como el modelo corpuscular de la materia, el modelo de interacciones mecánicas, el modelo de cambio químico o el modelo de ser vivo, entre otros. Se refieren a "las ideas básicas, fundamentales, irreducibles, que las ciencias han establecido para pensar sobre los diferentes tipos de fenómenos que estudian (químicos, genéticos, ecológicos, tectónicos, mecánicos, electromagnéticos...)" (Izquierdo y Adúriz-Bravo, 2005). Aplicando estos modelos escolares clave a diferentes fenómenos se construyen otros modelos científicos escolares también objeto de enseñanza, como el modelo de gas ideal, el de péndulo simple, el de reacción de combustión, o el de ser vivo autótrofo. El modelo de energía ${ }^{1}$ aquí expresado es para nosotros un modelo científico escolar clave, de naturaleza teórica, que sirve para describir, predecir, explicar e intervenir en el mundo desde la mirada energética, de forma coherente con el conocimiento científico erudito, pero que constituye nuestra forma de aproximarnos al mismo en ciencia escolar.

El proceso de construcción de modelos es lo que se denomina modelización (Justi, 2006; Schwarz y Gwekwerere, 2007; Windschitl Thompson y Braaten, 2008; Hernández et al., 2015; Couso y Garrido, 2016). Coincidimos con Schwarz y colegas en que la modelización debe entenderse como el proceso, tanto personal como social, de "ir dando sentido a las ideas en desarrollo" (Schwarz et al. 2009: 637). Existe un consenso amplio en la literatura sobre modelización respecto al hecho de que modelizar implica que los estudiantes sean capaces no sólo de construir y utilizar modelos, sino sobre todo de evaluarlos y revisarlos (Khan 2007; Clement 2008; Schwarz et al. 2009). A diferencia de estos autores, sin embargo, nuestra propuesta considera la construcción de modelos cada vez más sofisticados como la actividad esencial de la modelización (y no una etapa inicial de la misma). Esta construcción paulatina requiere la participación de alumnos y alumnas en las prácticas de expresión explícita de sus modelos mentales, uso de sus modelos para predecir o explicar fenómenos, evaluación de sus modelos en base a las pruebas disponibles y revisión de sus modelos en base a nuevas ideas (Couso y Garrido, 2017; Garrido 2016). Para guiar este proceso en el aula, las autoras proponen un ciclo de instrucción coherente con la literatura en el ámbito y el ciclo de aprendizaje.

Modelizar fenómenos para apropiarse de un modelo científico escolar implica poner a los estudiantes en situación de construir sus propias versiones de las ideas centrales de las disciplinas científicas, a través de articular su propia comprensión de cómo se comporta un fenómeno científico (Windschitl et al., 2008). Este proceso no sólo favorece el aprendizaje de las ciencias (entendido como la evolución de los modelos mentales de los alumnos), sino también el aprendizaje sobre ciencias. La necesidad de que la enseñanza de las ciencias aborde aspectos sobre su naturaleza se discute desde hace más de 100 años, y ha adquirido un importante consenso en la última década (Rudolph, 2005; OCDE, 2016). Cómo conseguirlo, sin embargo, es aún hoy objeto de acalorado debate. Una forma de lograr este objetivo que está cobrando importancia recientemente es la propuesta de aprender ciencia y sobre ciencia participando en las prácticas de construcción de conocimiento científico: modelización, indagación e

\footnotetext{
${ }^{1}$ A pesar de que otros autores han discutido la energía más como concepto estructurante entre diferentes disciplinas que como modelo en sí mismo, hablamos de "modelo de energía" por brevedad para referirnos a las ideas, reglas del juego y forma de mirar que queremos enseñar sobre la energía.
} 
argumentación (Crujeiras y Jiménez-Aleixandre, 2012; Osborne, 2014). Al poner a los alumnos en situación de participar en procesos de creación de conocimiento análogos a los de la ciencia, el enfoque tradicional del aula de ciencias cambia de "enfoque en los productos" (teorías, leyes...) a "enfoque en los procesos". Con ello, se posibilita vivenciar y reflexionar sobre la ciencia como una forma de conocer además de un cuerpo de conocimientos (Duschl y Grandy, 2008; NRC, 2012). En este contexto la modelización es una práctica científica escolar clave, debido a que una visión epistemológicamente adecuada sobre cómo se construye el conocimiento científico debe estar más centrada en el papel de la teoría, la explicación y los modelos que en el generalmente estereotipado método científico (NRC, 2007; Windschitl et al., 2008).

\section{Progresar modelizando diferentes versiones del modelo científico escolar}

En paralelo a la apuesta por desarrollar actividades de modelización en la escuela para construir modelos, se plantea la necesidad de la enseñanza de ideas científicas de forma gradual y progresiva a lo largo de la escolaridad. Esta idea es especialmente potente en la modelización, porque la evidencia nos muestra que la enseñanza de ideas o conceptos de forma fragmentada no permite la abstracción de las ideas, reglas del juego y formas de mirar de los modelos científicos escolares (Couso, 2014), por lo que estas ideas deben estar ahí, en una versión sencilla pero potente, desde el inicio. Dicho de otro modo, la enseñanza de los modelos debería pensarse como una auténtica progresión de aprendizaje de los mismos, es decir, como un camino que permita irlos construyendo en aproximaciones sucesivas a lo largo de la escolaridad (Couso, 2014). Esta noción se recoge en la literatura de las progresiones de aprendizaje, entendidas como trayectorias probables en la evolución de la comprensión de un concepto o idea (Corcoran, Mosher y Rogat, 2009). Tanto en su formato teórico como empírico, las progresiones de aprendizaje han surgido como un apoyo al proceso de enseñanza en ciencias (Duschl et al., 2011). En su versión curricular, por ejemplo, proporcionan a los docentes un marco de referencia respecto que versión de cada concepto se debe trabajar con los estudiantes en cada nivel o estadio.

Existe en la literatura una enorme variedad de usos, formatos y métodos para construir progresiones de aprendizaje. En la enseñanza de modelos, una progresión útil incluye la descripción de las formas de entender, progresivamente más sofisticadas, de un modelo científico escolar. En las progresiones teóricas el límite superior se corresponde con la versión esperada del modelo científico escolar, o modelo objetivo (target model). Por ejemplo, sería explicar un fenómeno energético usando las ideas, reglas del juego y forma de mirar que expresamos en la Tabla 1. El límite inferior de la progresión representa la comprensión básica de las ideas del modelo, tal y como se identifica empíricamente en las ideas de los alumnos o se propone teóricamente des del conocimiento didáctico. Entre ambos límites se pueden identificar fronteras conceptuales (Zabel y Gropenhiesser 2011), cruzar las cuales implica superar un obstáculo conceptual importante y apoyarse en una nueva idea con potencialidad de sofisticación. La superación o no de estos obstáculos y los matices en el grado de apropiación de otros aspectos del modelo científico escolar definen estadios, niveles o peldaños intermedios en la progresión. Mediante la acción educativa se espera que los modelos mentales de los estudiantes, que interpretamos de su discurso, producciones y acciones, evolucionen en su grado de sofisticación. La progresión teórica ha de poder guiar esta evolución, mientras que la progresión empírica la ha de describir y analizar.

En el caso concreto de la energía, Neumann et al. (2013) realizaron un estudio de las progresiones de aprendizaje de energía de una muestra de estudiantes, y dentro de los 
principales hallazgos destacan que la menor dificultad presente en los estudiantes es identificar aspectos en torno a ideas relativas a las formas y fuentes de energía, mientras que las ideas en relación a la conservación de energía tienen un grado de dificultad alta, incluso mayor que las de degradación de la energía (tal y como Solomon ya propuso hace más de 50 años, Solomon 1982; 1985). Esta progresión permite establecer un orden recomendable para la enseñanza y aprendizaje del modelo de energía, usado en la Tabla 1.

\section{Preguntas de investigación}

En lo anterior hemos compartido una cierta perspectiva sobre los modelos y la modelización en la enseñanza de las ciencias, así como ideas sobre la enseñanza y aprendizaje de la energía, que son la esencia de nuestro trabajo de investigación en éste ámbito. Sin embargo, desde una visión de la Didáctica de las Ciencias al servicio de la mejora educativa y la perspectiva de la investigación basada en el diseño (DBRC, 2003) que compartimos, nuestro interés reside en operativizar estas ideas en alternativas de diseño y constructos didácticos de utilidad en el aula. En este sentido nos planteamos identificar los estadios que caracterizan la versión del modelo de energía de los estudiantes al final de su escolarización obligatoria, las fronteras conceptuales que estos cruzan al sofisticar su modelo de energía y la influencia que la calidad del diseño didáctico tiene en esta evolución. En consecuencia, nos planteamos las siguientes preguntas de investigación:

- ¿cómo progresan los estudiantes, de 40 de ESO desde sus modelos iniciales de energía hacia el modelo científico escolar que se espera construir con la implementación de un diseño didáctico centrado en la modelización?

- ¿cuáles son las fronteras conceptuales que deben cruzar los estudiantes para adquirir un modelo de energía más sofisticado?

- ¿qué influencia tiene en el progreso del modelo de energía de los estudiantes la calidad del diseño didáctico?

\section{Metodología}

\section{Contexto de la investigación}

Para responder a estas preguntas de investigación analizamos las respuestas de los estudiantes en el marco de un taller experimental especialmente diseñado para promover actividades de modelización del modelo científico escolar de energía. El taller experimental "Disipación de la energía por rozamiento" es uno de los talleres ofrecidos en el programa REVIR ${ }^{2}$ de la Universidad Autónoma de Barcelona (UAB), realizados en un laboratorio informatizado y dinamizado por monitores expertos en didáctica de las ciencias. El taller analiza los cambios en el estado de un sistema concreto (una rueda) entre su puesta en movimiento por acción manual y el reposo que se produce al frenarla, y propone seguir las transferencias y disipaciones de energía midiendo la temperatura de una pequeña placa de cobre situada exactamente entre la pastilla de freno y la rueda, que se calienta al accionar el freno y se enfría al cabo de un rato (López y Pintó, 2012b).

\footnotetext{
2 El programa REVIR es un proyecto de colaboración entre el centro de investigación CRECIM y la Fundación Bancaria La Caixa. Más información en: http://crecim.uab.cat/revir/
} 
El taller se implementó en tres momentos del curso 2012-13, con una participación total de 68 estudiantes. Después de cada una de las tres implementaciones se identificó la necesidad y llevaron a cabo diferentes cambios didácticos (refinamientos del diseño), producto tanto del análisis de las respuestas de los estudiantes como de las reflexiones de los investigadores.

\section{Recogida de datos}

Los datos usados en la investigación corresponden a las producciones escritas de estudiantes de tres grupos clase diferentes en el contexto del taller experimental descrito. Al inicio y al final del taller se propusieron dos preguntas distintas pero substancialmente equivalentes, ambas en referencia al camino de la energía (ver Tabla 2).

Tabla 2. Pregunta inicial y final inseridas en el taller experimental

\begin{tabular}{|l|l|l|}
\hline Contexto & \multicolumn{1}{|c|}{ Situación inicial } & $\begin{array}{l}\text { La iluminación por incandescencia de } \\
\text { un disco de freno en un coche Rally. }\end{array}$ \\
\hline Pregunta & $\begin{array}{l}\text { El calentamiento de una placa de cobre } \\
\text { que actúa de freno de una rueda en } \\
\text { movimiento. }\end{array}$ \\
\hline $\begin{array}{l}\text { Explica de donde proviene y qué } \\
\text { camino ha seguido la energía asociada } \\
\text { a la luz del disco de freno, desde que } \\
\text { la rueda está en movimiento hasta } \\
\text { que el coche está parado y el disco } \\
\text { deja de brillar. }\end{array}$ & $\begin{array}{l}\text { ¿Serías capaz de describir el camino de de } \\
\text { la energía desde que hemos puesto la } \\
\text { rueda en funcionamiento hasta que la } \\
\text { placa de cobre se ha enfriado por } \\
\text { completo? }\end{array}$ \\
\hline
\end{tabular}

\section{Análisis de las respuestas de los estudiantes}

Las producciones de los estudiantes en las preguntas de la Tabla 2 fueron analizadas de forma cualitativa-interpretativa, categorizando las diferentes ideas de los estudiantes de acuerdo a los aportes teóricos (por ejemplo las ideas del MCE de energía de la Tabla 1 o las ideas alternativas de los estudiantes en el tema) pero también de los datos (por ejemplo categorías sugeridas por la forma particular de entender la energía de estudiantes concretos). Así, se ha utilizado un enfoque combinado de análisis deductivoinductivo para la identificación de categorías (versiones del MCE de energía de los alumnos), que se han ido refinando según el Método de Comparación Constante (Huberman y Miles, 2002) hasta su saturación. Estas categorías se presentan en la Tabla 3.

Las dos primeras categorías de la tabla 3 corresponden a las respuestas de los alumnos que no se realizan en términos energéticos, siendo $\mathrm{P}$ la categoría para les explicaciones que se centran en procesos, observables u objetos, sin asociarlos a cambios ni a agentes de cambio, y $\mathrm{M}$ las explicaciones donde los estudiantes asocian los cambios a un agente mecánico como la fricción. En cambio, las cuatro categorías posteriores ( $T$, WQ0, WQ1, WQ2/WQ3) corresponden a las respuestas de estudiantes que sí incluyen aspectos 
energéticos, y el orden ascendente corresponde al grado de sofisticación de estas respuestas. Así, los estudiantes situados en la lógica del estadio T explican en términos de transformaciones entre tipos de energía en lugar de en términos de transferencia, conservación o degradación de la energía. En el estadio WQO los estudiantes hablan de la transferencia de energía entre sistemas o partes de un sistema, pero sin mencionar a través de qué mecanismos ocurren dichas transferencias. El estadio WQ1 corresponde a las respuestas donde aparecen los mecanismos de transferencia trabajo y/o calor, y el estadio WQ2/WQ3 corresponde a las respuestas donde, además, aparece la idea de degradación de la energía, y en algún caso puntal, la idea combinada de degradación y conservación.

Tabla 3. Estadios, descripción y ejemplo de respuesta para cada uno

\begin{tabular}{|c|c|c|}
\hline Código & $\begin{array}{c}\text { Descripción del estadio al que se asocia } \\
\text { la explicación }\end{array}$ & $\begin{array}{c}\text { Ejemplo (código del estudiante y cita } \\
\text { textual) }\end{array}$ \\
\hline $\mathrm{P}$ & $\begin{array}{l}\text { Describe los procesos, observables u } \\
\text { objetos sin explicar en términos ni } \\
\text { mecánicos ni energéticos. } \\
\text { Enumeran varios o todos los procesos, } \\
\text { observables u objetos que se identifican } \\
\text { en el fenómeno, sin asociarlos a energía } \\
\text { ni a una causalidad. }\end{array}$ & $\begin{array}{l}\text { Estudiante A16: "Movimiento, fricción } \\
\text { del freno, aumenta la temperatura } \\
\text { (calentamiento), disminución de la } \\
\text { temperatura (enfriamiento) para llegar a } \\
\text { la temperatura ambiente }\left(\mathrm{T}_{\mathrm{o}}\right)^{\prime} \text {. }\end{array}$ \\
\hline $\mathrm{M}$ & $\begin{array}{l}\text { Explica en términos de interacción } \\
\text { mecánica pero no en términos de } \\
\text { energía. } \\
\text { Identifica el agente de cambio (por } \\
\text { ejemplo, la fricción) como causa de los } \\
\text { cambios observados. En este estadio los } \\
\text { estudiantes pueden o no identificar } \\
\text { observables, explicar a niveles } \\
\text { microscópico o macroscópico e incluso } \\
\text { hacer referencia a la energía, pero como } \\
\text { algo generado por el agente de cambio. }\end{array}$ & $\begin{array}{l}\text { Estudiante A15: "La rueda al fregar con el } \\
\text { cobre hace que las partículas choquen y } \\
\text { se muevan haciendo que la temperatura } \\
\text { aumente y pero cuando la rueda para, el } \\
\text { cobre se nivela con la temperatura } \\
\text { ambiente". }\end{array}$ \\
\hline $\mathrm{T}$ & $\begin{array}{l}\text { Explica en términos de transformación } \\
\text { de la energía. } \\
\text { Describe el fenómeno en términos } \\
\text { predominantemente energéticos, pero } \\
\text { mencionando tipos de trasformaciones } \\
\text { de energía, asociándoles un nombre } \\
\text { (energía cinética, energía calorífica, } \\
\text { energía luminosa, etc.). En algunos casos } \\
\text { puede asociar la energía a procesos u } \\
\text { observables, pero no identifica } \\
\text { transferencias de energía de un sistema } \\
\text { a otro, ni los mecanismos por los que se } \\
\text { transfiere. }\end{array}$ & $\begin{array}{l}\text { Estudiante B11: "La energía proviene del } \\
\text { coche, el coche al frenar hace una fuera } \\
\text { de fricción la que afecta al disco de } \\
\text { frenos, la energía pasa a ser calorífica y } \\
\text { luminosa, el disco de frenos se calienta y } \\
\text { se ilumina". }\end{array}$ \\
\hline WQO & $\begin{array}{l}\text { Explica en términos de transferencia de } \\
\text { energía de un sistema a otro. } \\
\text { El estudiante demuestra tener una idea } \\
\text { de transferencia de energía de un } \\
\text { sistema a otro, pero no menciona los } \\
\text { mecanismos por los que ésta se } \\
\text { transfiere. }\end{array}$ & $\begin{array}{l}\text { Estudiante B8: "Primero transmitimos la } \\
\text { energía desde nuestro brazo a un } \\
\text { cuerpo, este cuerpo es la rueda. Después } \\
\text { este cuerpo al ser frenado por el cobre } \\
\text { ha transmitido una parte de la energía de } \\
\text { nuestro brazo al cobre y otra parte a la } \\
\text { misma estructura del cuerpo, finalmente } \\
\text { al cobre y del mismo cuerpo al entorno". }\end{array}$ \\
\hline
\end{tabular}




\begin{tabular}{|c|c|c|}
\hline WQ1 & $\begin{array}{l}\text { Explica en términos de transferencia de } \\
\text { energía de un sistema a otro } \\
\text { mencionando mecanismos (calor y } \\
\text { trabajo). } \\
\text { El estudiante es capaz de asociar los dos } \\
\text { mecanismos de transferencia de energía } \\
\text { o bien uno de los dos, relacionándolos } \\
\text { con una fuerza (en el trabajo) o un con } \\
\text { una diferencia de temperatura (en el } \\
\text { calor). }\end{array}$ & $\begin{array}{l}\text { Estudiante A9: "Hay energía cinética, } \\
\text { cuando se produce la fricción entre el } \\
\text { cobre y la rueda se trata de un trabajo, } \\
\text { cuando se enfría el cobre se trata de } \\
\text { trasmisión de energía por calor al } \\
\text { entorno". }\end{array}$ \\
\hline $\begin{array}{l}\text { WQ2/ } \\
\text { WQ3* }\end{array}$ & $\begin{array}{l}\text { Explica en términos de transferencia, de } \\
\text { degradación (* y de conservación) de } \\
\text { energía. } \\
\text { El estudiante es capaz de asociar los dos } \\
\text { mecanismos de transferencia de energía } \\
\text { o bien uno de los dos, relacionándolos } \\
\text { con una fuerza (en el trabajo) o un con } \\
\text { una diferencia de temperatura (en el } \\
\text { calor). Además, identifica la degradación } \\
\text { de la energía relacionándola con la } \\
\text { disipación, la utilidad o la calidad de la } \\
\text { energía a lo largo de una cadena } \\
\text { energética. }\end{array}$ & $\begin{array}{l}\text { Estudiante B3: "La energía comienza en } \\
\text { el brazo de la persona, seguidamente es } \\
\text { transmitida gran parte de ésta a la rueda } \\
\text { (trabajo) y una pequeña parte al } \\
\text { ambiente. Después una parte de la } \\
\text { energía que tenía la rueda es transmitida } \\
\text { al cobre y otra parte al ambiente. } \\
\text { Finalmente, la energía acaba toda en el } \\
\text { ambiente ya que el cobre se ha enfriado } \\
\text { (transferencia por calor al entorno), } \\
\text { pierde su utilidad". }\end{array}$ \\
\hline \multicolumn{3}{|c|}{$\begin{array}{l}\text { * La idea de conservación de la energía no se refleja explícitamente en ninguna de las respuestas } \\
\text { de la pregunta inicial y final analizadas, pero sí aparece en otras actividades del taller para } \\
\text { algunos alumnos puntuales. }\end{array}$} \\
\hline
\end{tabular}

\section{Representación gráfica de las respuestas}

Una vez categorizadas las respuestas inicial y final de cada estudiante, realizamos una representación gráfica inspirada en la lógica de las progresiones de aprendizaje para la identificación de estadios (Duschl et al., 2011) y en la propuesta de Zabel y Gropengiesser (2011) para la señalización de fronteras conceptuales, tal como presentamos en la Figura 1. Los estadios aparecen representados a lo largo de una escalera, y las flechas curvas representan la trayectoria de cada uno de los estudiantes desde el estadio inicial al principio del taller hasta el estadio en el que se sitúa al final. El código de colores verde, naranja y rojo de las flechas corresponde a los casos en que se aumenta, se mantiene o se reduce el grado de sofisticación en la versión del MCE de energía del alumno entre ambas respuestas. Además, hemos representado el código de estudiante asociado en el vértice de cada parábola, con el grosor de las flechas representando el número de estudiantes.

En la representación, las líneas discontinuas entre los estadios $P, M$ y $T$, de mayor grosor, corresponde a las dos principales fronteras conceptuales identificadas entre maneras de explicar el fenómeno (procesos, aspectos mecánicos y aspectos energéticos), mientras que las líneas de menor grosor superiores dividen los estadios que se sitúan en una lógica energética (ver ejemplos Tabla 3). 


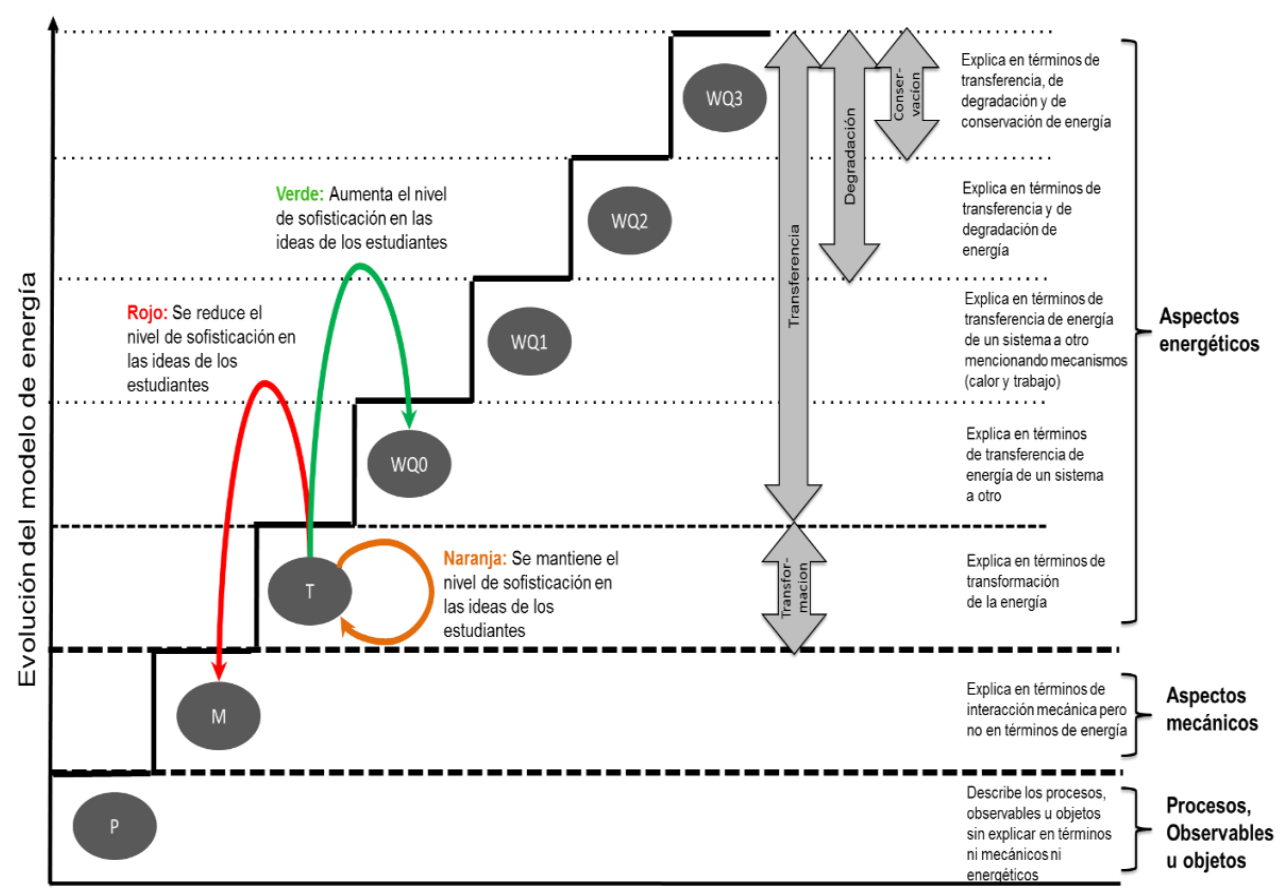

Figura 1. Gráfico de evolución del modelo de energía de los alumnos a lo largo de una intervención

\section{Resultados}

El análisis de las respuestas de los estudiantes correspondientes a la primera y tercera implementación del taller (con dos refinamientos didácticos de por medio) permite obtener dos gráficos de evolución del modelo de energía de los estudiantes a lo largo del mismo (Figura 2).

En la primera implementación con estudiantes de 4ㅇ de ESO (A1-A24) se puede observar que el punto de partida o modelo inicial de los estudiantes está básicamente en los estadios no energéticos $P$ y $M$, con algún estudiante en explicando en términos de transformación T (escalera de la izquierda). A lo largo de esta implementación 11 estudiantes avanzan de sus modelos iniciales a versiones más sofisticadas de éste, 6 estudiantes se mantienen en el modelo en el que empezaron y 7 retroceden. El mayor avance entre estadios lo encontramos en el estudiante A18, que pasa de explicar que " $E I$ rozamiento de las pastillas calientan el disco, luego la energía cinética, la llanta se calienta y como es metálica se vuelve de color rojo" a explicar que "Aplico una fuerza -> energía cinética, se produce la fricción entre el cobre y la rueda a través de un trabajo -> el cobre se enfría (transmisión de la energía por calor)". En este ejemplo se puede observar cómo se pasa de usar el término "calentar" como sinónimo de aumentar la temperatura al término "calor" como mecanismo de transferencia asociado a la diferencia de temperatura entre dos cuerpos. 


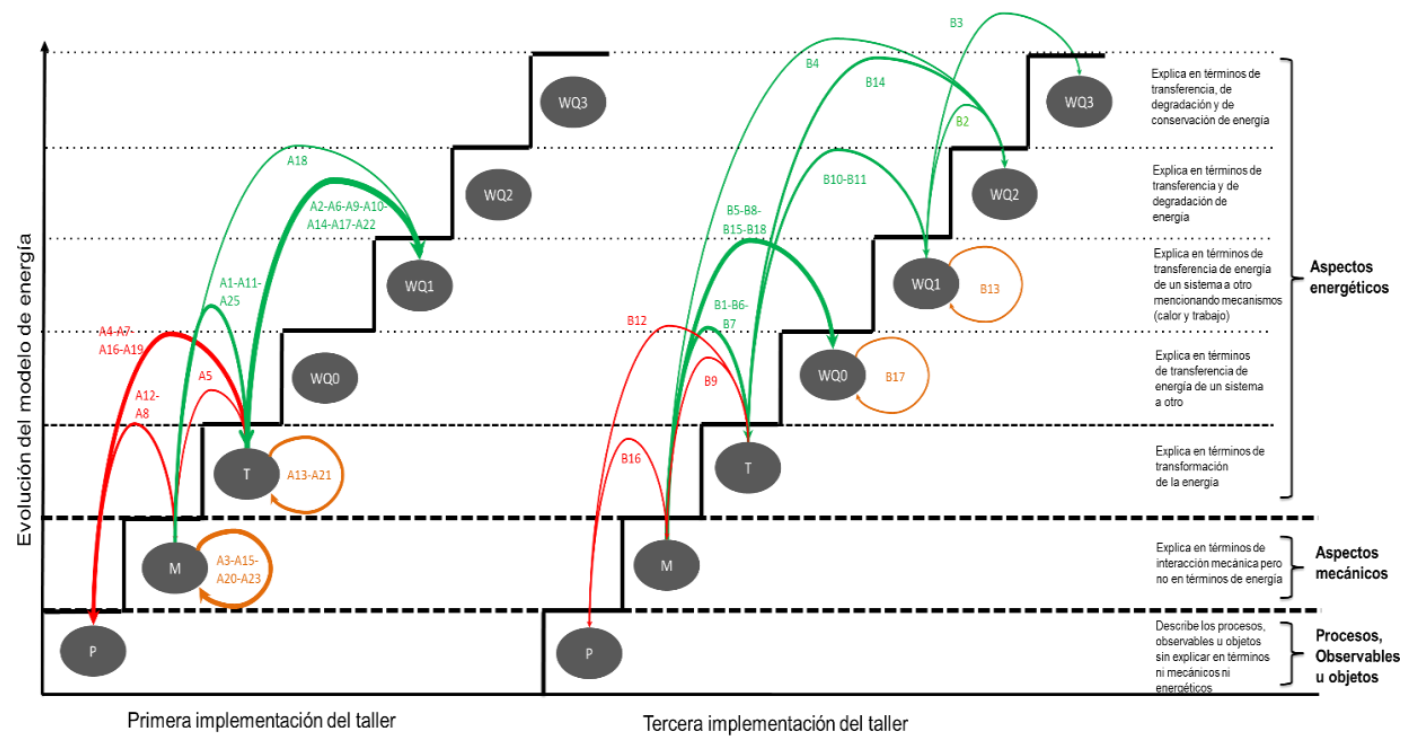

Figura 2. Evolución de los estadios de energía de estudiantes de 4ㅇ de ESO, primera implementación (izquierda) y tercera implementación (derecha)

La escalera de la parte derecha de la Figura 2 representa la evolución en los estadios del modelo energético en la tercera implementación del taller, después de dos refinamientos del mismo, en otro grupo de estudiantes de 4을 de ESO (B1-B18). De nuevo el punto de partida de la mayoría de los estudiantes se encuentra en los estadios sobretodo pre-energéticos y de transferencia, hay tres alumnos que parten de modelos de energía en estadios superiores. En esta tercera implementación del taller se observa que 13 estudiantes cambian a una explicación correspondiente un estadio superior, 3 estudiantes bajan a un estadio inferior y 2 se mantienen en el mismo estadio. Interesantemente 5 de ellos terminan incluyendo en sus respuestas aspectos de los estadios superiores del modelo de energía (degradación). Por ejemplo, el estudiante B4 se desplaza de un estadio $\mathrm{M}$ inicial en el que explica "el color rojizo proviene de la energía generada al frenar y del rozamiento entre el disco de frenos y las pastillas" a un estadio WQ2, explicando: "La energía potencial asociada al hombre se transfiere a la rueda por trabajo, la que adquiere energía cinética y al rozar con el cobre gana temperatura, debido al trabajo, que luego se disipa al ambiente cuando el cobre se enfría, a través de calor".

Si comparamos ambas escaleras de la Figura 2, vemos que en la primera implementación ningún estudiante se apropia de un modelo altamente sofisticado, en términos de degradación y conservación de la energía, mientras esto sí sucede en la tercera implementación. Comparando el sentido, origen y destino de las flechas podemos observar que la cantidad (11/24 vs $13 / 18)$ y calidad de los ascensos también es superior en la tercera implementación. También se puede observar que el número de estudiantes que retrocede a estadios inferiores es también proporcionalmente menor $(7 / 24$ vs $3 / 18)$. Finalmente se observa que los estudiantes que se mantuvieron en un mismo estadio en la tercera implementación partían ya de un modelo bastante sofisticado.

El análisis de las fronteras conceptuales cruzadas por los estudiantes en las dos implementaciones (ver Figura 2) nos muestra también importantes diferencias entre ambas. En la primera implementación más de la mitad de los estudiantes (un 54\%) supera la principal frontera perseguida con la actividad del taller, pasando de no usar un 
modelo de energía para explicar fenómenos del mundo a usarlo. Por otro lado, sólo un $33,3 \%$ se sitúa en un estadio que podemos considerar cercano al modelo escolar de energía (estadios WQ), utilizando ideas de transferencia, conservación o degradación que superan una mera visión de transformación de la energía (estadio T). Este resultado muestra la dificultad que existe para superar la lógica basada en la idea de transformación de la energía y los tipos de energía, tan común también en los propios recursos educativos), y que la literatura discute como altamente persistente si no se trabaja en profundidad la idea de transferencia de energía (Neumann et al, 2013).

En el caso de la tercera implementación el resultado más relevante es que prácticamente todos los alumnos finalizan el taller hablando en términos de energía (84\%) y hasta un $67 \%$ supera la importante "barrera" de explicar en términos de transformación (estadio T) y consigue hacerlo en términos de transferencia (estadios WQ). Es decir, en el momento final la mayoría de los estudiantes se sitúa en estadios que consideramos adecuados desde el modelo escolar de energía.

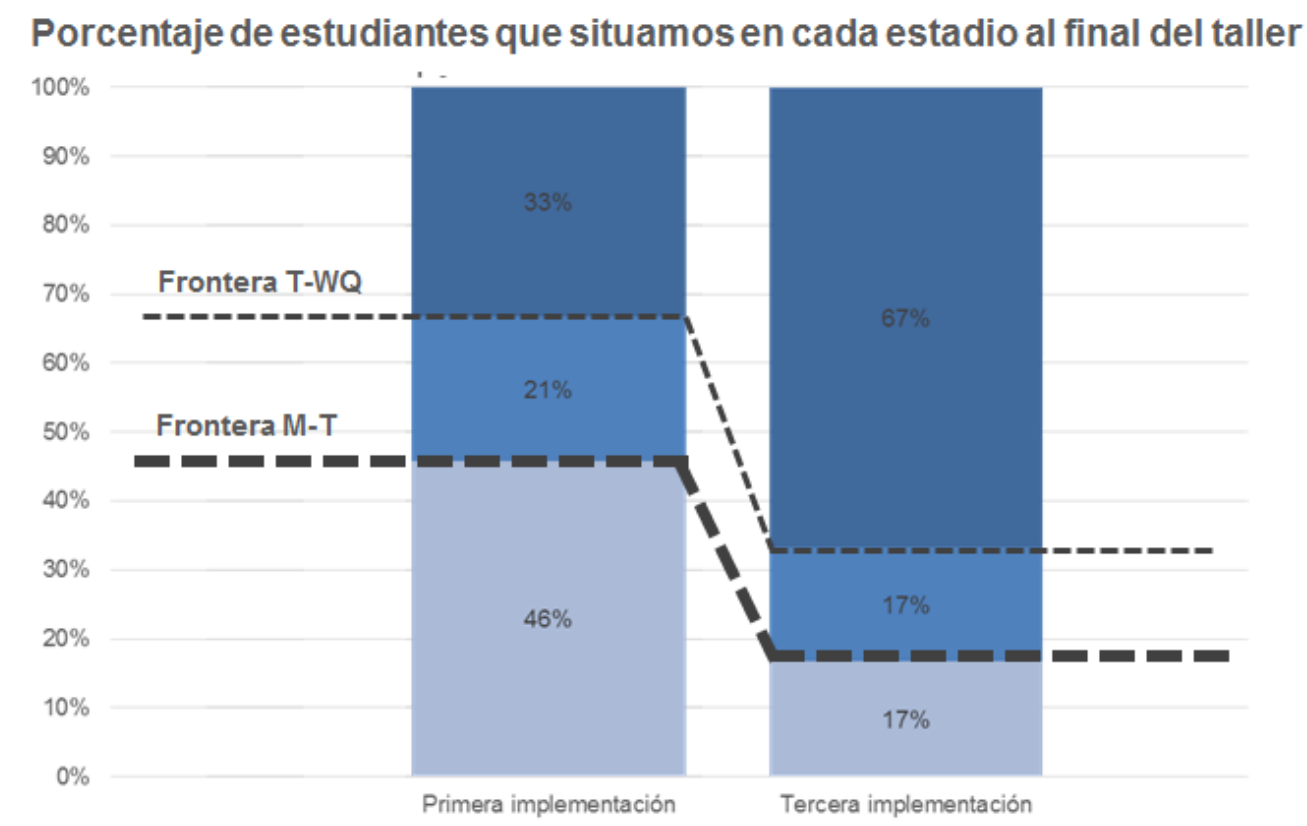

Figura 3. Porcentaje de alumnado que supera las diferentes fronteras conceptuales del MCE de energía

La comparación entre las implementaciones del taller inicial y final nos permite analizar la posible influencia del diseño didáctico, y en concreto de los refinamientos realizados, en la evolución de los modelos de energía de los alumnos. A pesar de que ambos grupos de estudiantes no son necesariamente comparables entre sí (más allá de que son de institutos similares en nivel socio-económico y del mismo curso escolar), y a pesar del hecho de que los puntos de partida son ligeramente mejores en el segundo caso para tres estudiantes, la importancia de la mejora obtenida no puede atribuirse solamente a esta razón. La principal explicación del cambio en el tipo de evolución ocurrida entre ambas implementaciones lo atribuimos, en gran medida, a la mejora en el diseño didáctico del taller entre la primera y la tercera implementación.

El análisis de las respuestas de los estudiantes en la primera implementación situaba a la mayoría del alumnado en modelos pre-energéticos o sólo transformativos, lo que sugería importantes limitaciones del diseño didáctico a confrontar. Una revisión 
reflexiva por parte de los investigadores permitió identificar aspectos a refinar, en particular respecto a las ideas de conservación y degradación de la energía. Estas no se trabajaban ni tan explícitamente, ni con el mismo nivel de actividad, discusión y tiempo adjudicado que la idea de transferencia. Ejemplos de estos refinamientos fueron la introducción de la pregunta "¿de qué otras maneras se podría aprovechar la energía?" para cada etapa del camino de la energía en el fenómeno (antes que la rueda girara, mientras la rueda giraba, después de frenar, etc.). Esto permitió incidir en la idea que la energía, a pesar de conservarse, era cada vez menos útil. Otro cambio destacado en el taller fue pedir a los estudiantes que escogieran explícitamente, para cada transferencia en cada etapa del camino de la energía, qué tipo de mecanismo podía explicarlo (trabajo o calor). Estos y otros ejemplos de cambios realizados en el diseño didáctico del taller tienen en común que en ningún caso se modificó el montaje experimental, ni el sistema de recogida de datos (el sensor de temperatura), ni la representación de los datos experimentales obtenidos por los estudiantes, sino la manera de promover que los estudiantes pensaran usando las ideas del modelo escolar de energía (transferencia, degradación y conservación). La guía didáctica completa se encuentra disponible online en López, Couso y Pintó (2016), donde también se incluye una discusión en mayor detalle de la versión final del diseño didáctico.

\section{Conclusiones}

Los estadios y las fronteras conceptuales del modelo de energía identificados en nuestros alumnos al final de su escolaridad son compatibles con la literatura sobre progresiones de aprendizaje de energía descritas por otros autores (Neumann et al. 2013), y matizan sus resultados sobre todo respecto a la existencia de estadios preenergéticos considerablemente presentes en las explicaciones iniciales del alumnado. Estos estadios y fronteras nos han ayudado a identificar las principales dificultades de los alumnos cuando se enfrentan a fenómenos reales y se les pide una explicación cualitativa de los mismos en términos energéticos. Además, la comparativa entre estadios finales y fronteras cruzadas por los alumnos nos ha servido para evaluar la calidad de la intervención educativa y orientar su refinamiento.

Las mejoras introducidas en el diseño a lo largo de dos ciclos de refinamiento, basadas tanto en el análisis de los modelos de los estudiantes como en una reflexión experta, permiten observar una importante evolución en los estadios del modelo de energía que usan los estudiantes en sus respuestas finales. Aunque sabemos que una mayor grado de sofisticación en las ideas del modelo que usan los estudiantes para elaborar sus respuestas no implica una apropiación total de las ideas del modelo, en particular respecto a su uso en otros contextos, los resultados muestran que 1. La propuesta didáctica diseñada tiene potencial para hacer mejorar los modelos de los alumnos y 2. La mejora basada en resultados de investigación de las propuestas didácticas es efectiva.

Es importante señalar, sin embargo, que este estudio e investigación presentan limitaciones tanto metodológicas como didácticas. En primer lugar, y como hemos dicho, hemos comparado dos grupos clase a priori comparables. Necesitaríamos (y en ellos estamos) más investigación con más estudiantes para poder concluir que el tipo de diseño didáctico que realizamos, basado en unas ideas particulares del modelo científico escolar de energía y en una forma de entender la modelización, son realmente efectivos. En segundo lugar, el aprendizaje aquí reportado está limitado por el alcance de una única intervención equivalente a 4 sesiones de aula, lo que señala la importancia 
de futuros trabajos en los que la intervención sea de mayor alcance y se evalúe también la capacidad de transferencia en el uso de un modelo sofisticado de energía a otros contextos. Por ejemplo, una secuencia didáctica más larga que trabaje con mayor profundidad los energy-related concepts debería permitir a los estudiantes realizar análisis de los fenómenos utilizando una mirada macro y micro de la energía.

Por último, y aunque no podamos concluir que los estudiantes participantes de esta investigación se hayan apropiado "de una vez y para siempre" del modelo escolar de energía esperado (ni siquiera aquellos que llegan al estadio superior), creemos que las experiencias de modelización que caminan en esta dirección desde la perspectiva de la práctica científica (altamente sociales y discursivas) posibilitan que los "pasos hacia atrás" en el modelo al enfrentarse a futuras situaciones sean más pequeños, y que el avance a estadios más sofisticados sea cada vez más rápido (Garrido, 2016).

\section{Agradecimientos}

Esta publicación ha sido parcialmente financiada por el Ministerio de Economía y Competitividad del gobierno español (EDU2015-66643-C2-1-P).

\section{Referencias bibliográficas}

Couso, D. (2014). De la moda de "aprender indagando" a la indagación para modelizar: una reflexión crítica. 26EDCE. Investigación y transferencia para una educación en ciencias: Un reto emocionante (pp. 1-28). Huelva: Servicio de Publicaciones Universidad de Huelva.

Couso, D. y Garrido, A. (2017) Models and modelling in elementary school pre-service teacher education: Why we need both. Cognitive and Affective Aspects in Science Education Research (pp. 263-280). Springer International Publishing: Helsinki.

Clement, J. J. (2008). Model Based Learning and Instruction in Science. En J. J. Clement y M. A. Rea-Ramírez (Eds.), Model Based Learning and Instruction in Science. The Netherlands: Springer.

Crujeiras, B. y Jiménez Aleixandre, M. P. (2012). Participar en las prácticas científicas: aprender sobre la ciencia diseñando un experimento sobre pastas de dientes. Alambique. Didáctica de las ciencias experimentales, 72, 12-19.

De Almeida Pacca, J. L. y Henrique, K. F. (2004). Dificultades y estrategias para la enseñanza del concepto de energía. Enseñanza de las ciencias, 22(1), 159-166.

Devi, R., Tiberghien, A., Baker, M., y Brna, P. (1996) Modelling students' construction of energy models in physics. Instructional Science, 24(4), 259-293.

Doménech, J. L., Limiñana, R. y Menargues, A. (2013). La superficialidad en la enseñanza del concepto de energía: una causa del limitado aprendizaje alcanzado por los estudiantes de bachillerato. Enseñanza de las Ciencias, 31(3), 103-119.

Driver, R. y Warrington, L. (1985). Students' use of the principle of energy conservation in problem situation. Physics Education, 20, 171-176.

Duit, R. (1981). Understanding Energy as a Conserved Quantity - Remarks on the Article by R. U. Sexl. European Journal of Science Education, 3(3), 291-301.

Duit, R. (1984). Learning the energy concept in school-em- pirical results from The Philippines and West Germany. Physics Education, 19, 59-66. 
Duschl, R. A. y Grandy, R. E. (2008). Teaching Scientific Inquiry: Recommendations for Research and Implementation. Rotterdam, Netherlands: Sense Publishers.

Duschl, R., Maeng, S. y Sezen, A. (2011). Learning progressions and teaching sequences: a review and analysis. Studies in Science Education, 47(2), 123-182.

García-Carmona, A. y Criado, A. M. (2008). Enfoque CTS en la enseñanza de la energía nuclear: análisis de su tratamiento en textos de física y química de la ESO. Enseñanza de las Ciencias, 26(1), 107-124.

García-Carmona, A. y Criado, A. M. (2010). La competencia social y ciudadana desde la educación científica: una experiencia en torno al debate de la energía nuclear. Investigación en la Escuela, 71, 25-38.

Garrido, A. (2016). La modelización y modelos en la formación inicial de maestros de primaria desde la perspectiva de la práctica científica. Tesis Doctoral, Universidad Autónoma de Barcelona.

Harlen, W. (2010). Principles and big ideas of science education. Association for Science Education.

Hernández, M. I., Couso, D., y Pintó, R. (2015). Analyzing Students' Learning Progressions throughout a Teaching Sequence on Acoustic Properties of Materials with a Model-Based Inquiry Approach. Journal of Science Education and Technology, 24(2-3), 356-377.

Justi, R. (2006). La enseñanza de ciencias basada en la elaboración de Modelos. Enseñanza de las ciencias, 24(2), 173-184.

Khan, S. (2007). Model-Based Inquiries in Chemistry. Science Education, 91(1), pp. 877905.

López, V. y Pintó, R. (2012a). Ensenyar energia a secundària. Revista recursos de física, 9.

López, V. y Pintó, R. (2012b). Hot brakes and energy-related concepts: is energy lost? Physics Education, 47(1), 38-43.

López, V., Couso, D, y Pintó, R. (2016). Dissipació de l'energia per fregament, seqüència didàctica per a l'estudi de l'energia. Barcelona: Publicacions REVIR.

Millar, R. (2005). Teaching about energy. York: University of York.

Neumann, K., Viering, T., Boone, W., y Fischer, H. (2013). Towards a Learning Progression of Energy. Journal of research in science teaching, 50(2), 162-188.

NRC. (2007). Taking Science to School: Learning and Teaching Science in Grades K-8. Board on Science Education, Center for Education. Division of Behavioral and Social Sciences and Education. Washington, D.C.: The National Academies Press.

NRC. (2012). A framework for K-12 Science Education. Practices, Crosscutting Concepts and Core Ideas. Washington, D.C.: The National Academies Press.

OCDE (2016) PISA 2015 Science Framework. En PISA 2015 Assessment and Analytical Framework. Science, Reading, Mathematic and Financial Literacy (pp. 17-46). Paris: OECD Publishing.

Ogborn, J. (1986). Energy and fuel -the meaning of «the go of things». En Driver R. y Millar, R. (eds.). Energy matters. University of Leeds. 
Oh, P. S. y Oh, S. J. (2011). What Teachers of Science Need to Know about Models: An overview. International Journal of Science Education, 33(8), 1109-1130.

Osborne, J. (2014). Teaching scientific practices: Meeting the challenge of change. Journal of Science Teacher Education, 25(2), 177-196.

Papadouris, N., Constantinou, C. y Kyratsi, T. (2008) Students' use of the energy model to account for changes in physical systems. Journal of Research in Science Teaching, 45(4), 444-469.

Pérez, M., Marbà, A., e Izquierdo, M. (2016). ¿Cómo se conceptualiza la energía en las unidades didácticas de biología? Enseñanza de las ciencias, 34(1), 73-90.

Pérez-Landazábal, M. y Varela Nieto, M. (2006). Una propuesta para desarrollar en el alumno de secundaria una visión unificada de la física a partir de la energía. Revista Eureka de sobre Enseñanza y Divulgación de las Ciencias, 3(2), 237-250.

Pintó. R. (1991). Algunos conceptos implícitos en la 1o y la 2o Leyes de la Termodinámica: una aportación al estudio de las dificultades de su aprendizaje. Tesis Doctoral, Universidad Autónoma de Barcelona.

Pintó, R., Couso, D. y Gutiérrez, R. (2005). Using research on teachers' transformations of innovations to inform teacher education. The case of energy degradation. Science Education, 89(1), 38-55.

Rudolph, J. L. (2005). Epistemology for the masses: The origins of the "scientific method" in American schools. History of Education Quarterly, 45(2), 341-376.

Schwarz, C. V. y Gwekwerere Y. N. (2007). Using a Guided Inquiry and Modeling Instructional Framework (EIMA) to Support Preservice K-8 Science Teaching. Science Education, 91(1), 158-186.

Schwarz, C. V., Reiser, B. J., Davis, E. A., Kenyon, L., Achér, A., Fortus, D., Shwartz, Y., Hug, B. y Krajcik, J. (2009). Developing a learning progression for scientific modeling: Making scientific modeling accessible and meaningful for learners. Journal of Research in Science Teaching, 46(6), pp. 632-654.

Solbes, J. y Tarín, F. (1998). Algunas dificultades en torno a la conservación de la energía. Enseñanza de las ciencias, 16(3), 387-397.

Solbes, J. y Tarín, F. (2004). La conservación de la energía: un principio de toda la física. Una propuesta y unos resultados. Enseñanza de las Ciencias, 22(2), pp. 185-294.

Solomon, J. (1982). How children learn about energy or does the first law come first? School Science Review, March 1982, 415 - 422.

Solomon, J. (1985). Teaching energy conservation. Physics Education, 20, 165-167.

Windschitl, M., Thompson, J. y Braaten, M. (2008). Beyond the scientific method: Model-based inquiry as a new paradigm of preference for school science investigations. Science Education, 92, 941-967.

Zabel, J. y Gropengiesser, H. (2011). Learning progress in evolution theory: climbing a ladder or roaming a landscape? Journal of biological education 45(3), 143-149. 